\title{
Ética empresarial y el desempeño laboral en Organizaciones de Alta Tecnología (OAT)
}

\author{
Ruiz C., Jocelyne K.* \\ Silva V., Neif G.** \\ Vanga A., María G. ***
}

\section{Resumen}

El presente artículo científico ofrece una visión integral del comportamiento de las variables, ética empresarial y el desempeño laboral en Organizaciones de Alta Tecnología (OAT). El propósito de la investigación fue determinar la relación entre la ética empresarial y el desempeño laboral, tomando como patrón las OAT. Así mismo, para obtener los resultados del estudio de tipo transeccional descriptivo, un instrumento de recolección de datos fue aplicado a una población constituida por 54 personas de distintas organizaciones del ramo tecnológico y que ocupan cargos gerenciales. Los resultados del estudio a pesar de que evidenciaron un alto nivel tanto en el desempeño laboral como en el cumplimiento de los códigos de ética por parte de los empleados, la relación entre las variables de investigación resultó ser positiva muy débil, lo que significa que mientras el empleado mejore el cumplimiento de la ética en sus labores, no necesariamente se evidenciará en él algún progreso del desempeño laboral. La investigación concluye sugiriendo una reorientación de los esfuerzos internos a fin de proporcionar al empleado una mayor participación a cursos de capacitación, talleres o charlas donde se compartan conocimientos entre los integrantes de la organización, todo esto planificado y organizado de acuerdo a las necesidades individuales y grupales. Asimismo, el desempeño de cada uno de los empleados de una OAT dependerá de la aplicación de verdaderos principios, valores y en explotar al máximo las propias capacidades, habilidades y conocimientos de modo que el conjunto de personas y entes a su alrededor pueda beneficiarse de ellas.

Palabras clave: Ética Empresarial, Desempeño Laboral, Organizaciones de Alta Tecnología.

Recibido: 10-06-07. Aceptado: 27-05-08

* Ingeniero en Computación, M.Sc. en Gerencia de Empresas. Profesora asistente adscrita al Departamento de Informática del Instituto Universitario de Tecnología de Maracaibo.

E-mail: jkruizc@gmail.com.

** Ingeniero en Computación. M.Sc. en Planificación y Gerencia. Profesor Asociado de la Facultad de Ciencias Económicas y Sociales de LUZ. E-mail: nsilva@luz.edu.ve

*** Ingeniero en Computación. M.Sc. en Gerencia Empresarial. Investigador docente del Instituto de Investigaciones de la Facultad de Arquitectura y Diseño de LUZ.

E-mail: mgvanga@yahoo.com. 
Ética empresarial y el desempeño laboral en Organizaciones de Alta Tecnología (OAT) Ruiz C., Jocelyne K.; Silva V., Neif G. y Vanga A., María G.

\title{
Business Ethics and Labor Performance in High Technology Enterprises (HTE)
}

\begin{abstract}
This scientific article provides a holistic vision of the behavior of business ethics and job performance variables in High Technology Organizations (HTO). The purpose of this study was to determine the relationship between business ethics and job performance, taking the HTOs as a pattern. Likewise, to obtain results from this of descriptive transectional-type study, a datacollecting tool was applied to a population made up of 54 people from different organizations in the technological branch who occupy management positions. Results showed that despite a high level in both job performance as well as compliance with the codes of ethics on the part of employees, the relationship between the research variables was a very weak positive, which means that while the employee improves in complying with ethics in his/her work, this does not necessarily produce any progress in job performance. The study concludes by suggesting a redirection of internal efforts in order to provide greater employee participation in training courses, workshops or lectures where knowledge is shared among the members of the organization, all of this planned and organized according to individual and group needs. Likewise, the performance of each employee in a HTO will depend on the implementation of true principles and values and on fully exploiting his/her own abilities, skills and knowledge so that the persons and entities around them can benefit from them.
\end{abstract}

Key words: Business ethics, job performance, High Technology Organizations.

\section{Introducción}

El tema de la ética de las organizaciones ha incrementado su vigencia, paralelamente, con el afianzamiento de la empresa en la sociedad contemporánea. Las empresas han alcanzado un posicionamiento clave porque son las generadoras de las riquezas además de ser el lugar que concentra un tiempo considerable de la gente y también el espacio esencial del aprendizaje de las personas, por lo que no existen acontecimientos en la sociedad que no tengan una directa y explícita relación con el mundo de las organizaciones.

A todo esto se agrega lo imposible que resulta no asociar la actividad humana con las connotaciones éticas; y en la actualidad ha renovado su vigencia dicho tema, toda vez que la complejidad del entorno así lo determinan.

Al respecto se puede indicar que si antes era importante tomar en cuenta este factor en el desenvolvimiento de las empresas, ahora es de suma urgencia. Aunque se ha visto que la ética es un asunto de principios, ahora es igualmente de naturaleza estratégica, dado que pudiera estar influenciando en los resultados provenientes del desempeño de cada uno de los integrantes de la organización, lo cual se traduce en el cumplimiento o no de los objetivos y metas de la misma.

Esta investigación se apoya en algunos modelos precedentes ya estudiados, pero insiste en la importancia de to- 
mar un rumbo nuevo dentro del ámbito de la ética, combinándola con el desempeño laboral en organizaciones de alta tecnología (OAT).

El propósito del presente artículo es determinar la relación entre la ética empresarial y el desempeño laboral, a través de lo cual se busca una manera de ofrecer oportunidades de crear, compartir y consultar información para reforzar los valores, creencias y principios; y al mismo tiempo facilitar a toda organización, alcanzar la ética empresarial deseada.

Esta investigación se clasifica por su propósito como aplicada, ya que está orientada a brindar solución a problemáticas que pudieran presentarse en organizaciones en relación a la ética de los empleados y su incidencia en el desempeño de sus labores. De acuerdo al método utilizado, la investigación se cataloga como descriptiva, y según el diseño, se considera no experimental, transeccional, correlacional-causal. La población estuvo conformada por 54 profesionales que desempeñan cargos gerenciales, en 8 empresas de alta tecnología del Estado Zulia, quienes respondieron dos (2) cuestionarios de escala tipo Lickert; uno para medir la variable ética empresarial y el otro para medir la variable desempeño laboral. Finalmente, Para calcular la relación entre la ética empresarial y el desempeño laboral, se recurrió a las estadísticas inferenciales a través de la correlación de Pearson.

Esta investigación se pudiera convertir en un camino que se promete fecundo para la sociedad en su conjunto y para los países en desarrollo en particular, orientándolos de una manera integral, alcanzar sus metas y objetivos propuestos.

\section{Etica empresarial}

La palabra Ética tal como es utilizada actualmente proviene de la palabra griega "ethos" que originalmente se refería a las prácticas y costumbres habituales. Es por ello que definir términos que tienen una larga historia no es sencillo; a lo largo de los años sus usuarios los han ido enriqueciendo con diferentes matices, e intentar ubicarlos en una definición resulta imposible.

Aún así, tomando en consideración esta advertencia, se puede señalar primeramente que "la ética es un tipo de saber de los que pretende orientar la acción humana en un sentido racional; es decir, pretende que se actúe racionalmente" (Cortina, 1998:17).

Por otra parte, Etkin (1993:16) afirma que la ética, "es el fundamento cuyos valores esenciales deben organizar la vida social, y son tales como la libertad y la dignidad humana, así como también se basa en conceptos morales como el bien común, lo bueno, lo equitativo y lo justo".

Analizando estas definiciones se denotan dos conceptos implicados: por un lado las relaciones interpersonales, que caracterizan a los individuos socialmente; y por el otro, un patrón de actuación deseable, donde existe una forma de conocimiento que establece un sistema moral, en la cual se distingue entre lo que se debe hacer y lo que no se debe hacer, elegir entre el vicio y la virtud, entre el bien y el mal.

La ética básicamente puede ser considerada como un saber netamente práctico, para actuar de un modo racional en la vida, consiguiendo de ella lo más posible, para lo cual es preciso saber or- 
Ética empresarial y el desempeño laboral en Organizaciones de Alta Tecnología (OAT) Ruiz C., Jocelyne K.; Silva V., Neif G. y Vanga A., María G.

denar las metas de la vida inteligentemente.

Es por ello que desde los orígenes de la ética, suele realizarse una primera distinción en el conjunto de los saberes humanos: los teóricos, preocupados por averiguar entre todo qué son las cosas, sin un interés explicito por la acción; y los prácticos, a los que importa discernir qué se debe hacer y cómo se debe orientar la conducta.

Asimismo, existen otras posiciones diversas en torno a la ética, abordando en primer lugar la opinión del padre de la ética utilitarista Bentham (2000), según su posición, la ética define lo bueno como aquello que produce placer y lo malo como aquello que produce sufrimiento. Por lo cual, el individuo persigue siempre su felicidad, para él lo bueno es aquello que produce felicidad y el deber del legislador es buscar la armonía entre el interés público y el privado. Agrega también, que el criminal no debe ser castigado con odio, sino sólo para prevenir el crimen, ya que es más importante que el castigo sea seguro a que sea severo.

Por otra parte, una segunda posición se puede tomar de otro precursor de la ética utilitarista Mills (2008), quien afirma que quienes pregonan la moral del sacrificio sólo buscan que otros se sacrifiquen por ellos, por lo que el orden moral es resultado de un equilibrio de intereses. En este sentido, los legisladores obran siempre en su propio interés y solamente el equilibrio de poderes y la opinión pública los orientan hacia el bien común. Además agrega que lo bueno es aquello que produce placer y esto se logra sólo mediante la satisfacción de una necesidad que puede ser de orden físico, emocional, afectivo, intelectual o espiritual. Los deseos pueden ser buenos o malos según acarreen o no la felicidad general. Por lo tanto la valoración de una acción depende primordialmente de sus consecuencias y no de la intención de la misma.

Este trabajo se apoya en la concepción de la ética utilitarista, por ser la que tiene mayor influencia en el pensamiento contemporáneo de nuestra sociedad y la filosofía del pensamiento de la empresa de tecnología sobre la cual se esta investigando. En este caso, la ética utilitarista puede verse justificada en el momento de obtener los resultados ya que las normas éticas se legitiman por su utilidad.

La finalidad de la ética utilitarista es mantener en marcha la tecnoestructura, es una ética aceptada por su eficacia. Su finalidad no es "realizar al hombre", ni "hacer mejores hombres". Su meta es optimizar el funcionamiento de los mecanismos políticos, económicos y sociales. Es por ello que el éxito de una empresa no está en proporción directa a su falta de ética. La existencia de organizaciones que pueden funcionar sin ética es algo muy relativo y cuestionable.

En este sentido es oportuno introducir el término ética empresarial, el cual según Ortiz (1995:18) "es un valor intrínseco de toda actividad económica y organizacional porque cualquier actividad empresarial atrae hacia sí un cúmulo de factores humanos", donde la dignidad personal es un valor que debe presidir las decisiones de cualquier organización y la convicción de que la armonía social exige la práctica habitual de conductas verdaderas.

Asimismo expone Pérez (1998: $32)$, que la ética empresarial es "el con- 
junto de conocimientos que ayudan a formular juicios acerca de las consecuencias de la aplicación de un plan de acción, en el plano del aprendizaje moral de lo afectado por el plan. En este sentido, en una afirmación de Cortina (1998), la ética empresarial se entiende como un cúmulo de valores, ideas morales y caracteres que persiguen los objetivos por los que la misma existe (satisfacer necesidades humanas).

Apoyando las definiciones anteriores, la ética empresarial tiene que ver con la conducta de los individuos que se desempeñan y dirigen las organizaciones, es el modo como se desarrollan los integrantes de empresas de acuerdo a las normas y patrones reconocidos y aceptados dentro del área organizacional y el ambiente socio-económico en el que se desenvuelven. Asimismo, es considerado anti-ético un comportamiento que no corresponda a ciertas normas deseadas o aceptadas dentro de la sociedad.

Según Fairkbanks (1999), en las OAT pareciera dejarse de lado la ética para poder hacer que la organización funcione. En estos casos la tecnología es el principal motor de cambio. Por ello, los líderes empresariales se dedican a reflexionar detenidamente sobre ella, a fin de administrarla de manera adecuada, no necesariamente atendiendo a los aspectos éticos que estas situaciones aparentan exigir.

En concordancia con lo anterior, exponen Silva y Espina (2006) que las Tecnologías de Información han posibilitado la renovación de procesos, nuevas formas de organización, nuevos modelos y cambios en la forma de trabajar repercutiendo sobre el mundo empresarial y siendo parte fundamental de los retos afrontados por la organización del siglo $X X I$ y la sociedad actual en la generación de nuevos paradigmas.

En el mismo orden de ideas, los autores afirman que la mayoría de las empresas del mundo en vías de desarrollo se muestran fascinadas ante la idea de adquirir tecnología para alcanzar una mayor competitividad y olvidan aspectos de post-adquisición que podrían diluir las ilusiones de convertirse en una organización competitiva.

En este sentido, es importante considerar que la tecnología puede imponer una gran tensión sobre los recursos escasos: humanos, técnicos, económicos y operativos, en conjunto con los aspectos éticos conforman los recursos integrales que al momento de adquirir tecnología deben tomarse en cuenta para lograr un mayor nivel de competitividad.

Según Cuesta (1998), existen dos razones por las cuales una OAT debe medir sus recursos integrales disponibles antes de adquirir una nueva tecnología para generar capacidades competitivas en la organización. La primera es que ninguna organización ni persona carece completamente de ética; y la segunda es que las empresas que programáticamente funcionan al margen de la ética, requieren de otras que sí funcionen éticamente.

Al respecto, actualmente se habla mucho de los valores éticos en el mundo empresarial de alta tecnología, más que en cualquier otra institución social, ya que estas se especializan en los procesos de producción y distribución de riqueza material, lo cual, es para gran parte de nuestra sociedad actual lo más importante.

Asimismo, en los procesos de direccionamiento empresarial, la fijación de 
Ética empresarial y el desempeño laboral en Organizaciones de Alta Tecnología (OAT) Ruiz C., Jocelyne K.; Silva V., Neif G. y Vanga A., María G.

valores éticos es una acción fundamental para guiar a todos sus integrantes en sus acciones laborales dentro de un contexto racional y moral. Es por ello que sería interesante discernir un poco sobre la ética empresarial y su relación con los valores y los principios.

\subsection{La ética empresarial y su relación con los valores y principios}

En este ámbito, la mayoría de las personas inmersas en una cultura determinada y en una época especifica, comparten valores; es decir, comparten un conjunto de normas fundamentales establecidas culturalmente para guiar las relaciones entre los individuos de la sociedad en la cual conviven, por lo que estarán de acuerdo en lo que ha sido establecido como "bueno" y como lo "malo".

De la misma forma, determinar que el comportamiento de un integrante de una organización es ético o no, depende en gran medida del sistema de valores de dicho individuo, ya que "son los criterios que guian la forma de actuación de las personas en forma individual o como integrantes de grupos, organizacionales y comunidades mayores" como lo expresa Etkin (1993:36).

En algunos casos, los valores son entendidos y usados como sinónimos de creencias y algunas veces visto como actitudes, por lo tanto es conveniente hacer un contraste entre estos términos. Según García y Dolan (1997) las creencias son consideradas como estructuras de pensamiento elaboradas y arraigadas a lo largo de la experiencia que sirven para explicar cómo es la realidad de los individuos y que se presentan antes de la configuración de los valores.
Por otra parte, las actitudes según Stoner (1994) son aspectos perdurables de la personalidad, resultantes de creencias y sentimientos acerca de algo en particular, de lo cual se derivan tendencias hacia un comportamiento positivo o negativo de ese algo. Según García y Dolan (1997) son consecuencia de los valores y las normas y pueden presentarse de manera positiva o negativa ante alguna situación o hecho.

Según lo discutido hasta ahora, las actitudes pueden ser consideradas como creencias con las mismas características intrínsecas de estas, las mismas se asemejan porque las actitudes son predisposiciones, las creencias son convicciones, ambas son afectivas, intelectuales y cambiantes, por lo que reflejan la posición de una persona y predicen la tendencia a actuar de determinada manera.

Según Siliceo y col. (1999), los valores, así como las actitudes y las creencias están unidos al comportamiento de los individuos y tienen mucha influencia en la forma como las personas perciben todo a su alrededor.

Por otra parte, apoyándose en las apreciaciones de Montaner (2001) los valores y las actitudes pueden ser considerados, entonces, como creencias que las personas aprenden a través de su vida, tomando en cuenta que los valores se dirigen más hacia la parte moral del individuo y las actitudes hacia el comportamiento que se presenta ante una cualquier situación.

En el mismo orden de ideas, Pérez (1998) afirma que los valores sociales de la mayoría de los miembros de la sociedad afectaran las creencias y las conductas de las personas. De la misma 
forma, los valores personales de la alta gerencia influencian las estrategias organizacionales; la actuación ética o antiética de un gerente es resultado de una interacción compleja entre la etapa de desarrollo moral del gerente, las distintas variables moderadoras que incluyen características individuales, el diseño estructural de la organización, la cultura organizacional presente y la intensidad del problema ético.

De lo anteriormente expuesto se puede inferir, que las personas carentes de un fuerte sentido moral son menos propensas a hacer cosas malas si se les restringe con reglas, políticas, descripciones de puesto o normas culturales poderosas que desaprueben tales comportamientos. Esta situación puede inclusive extenderse hasta corromper a individuos muy moralistas, dada la estructura y cultura organizacional que permite prácticas carentes de ética.

Es por ello que los valores determinan la creencia de una persona; y la ética el juicio de las obligaciones morales de las personas para con la sociedad a la cual pertenecen. En este sentido, es importante tener en cuenta que las normas morales van cambiando con el tiempo o época y esto dificulta la determinación precisa entre lo que es bueno y lo que es malo, por lo que grupos pertenecientes a una misma sociedad difieren o presentan ideas contradictorias sobre lo que está bien y lo que está mal.

Según todo lo que se ha planteado sobre valores, pueden entenderse como aprendizajes trascendentales relativamente estables en el tiempo que constatan que determinada forma de actuar es mejor que su opuesta para lograr que sal- gan bien las cosas. Al respecto, se puede afirmar que la estabilidad de los valores en el tiempo es relativa, ya que depende de la actitud del ser humano ante las creencias, principios y de su percepción ante las cosas. Así mismo la elección en la forma de actuación proviene de supuestos básicos o creencias sobre la naturaleza humana, dicho de otra forma, el ser humano elige pensar y actuar de un modo u otro, según crea que son las personas y las cosas.

En concordancia con lo expuesto, "el hecho de creer que no tener tiempo es signo de éxito en la vida" explica la relación entre las creencias y valores; esto contribuye a sustentar el valor del trabajo intenso. De esta forma, se observa la estrecha relación entre creencias y valores, y cuando se habla de cambios de valores en una organización, aún más si es una OAT, se deben hacer cambios en el aprendizaje de creencias para cambiar actitudes e influir positivamente sobre la conducta para obtener óptimos resultados.

Por otra parte, como lo afirma Leer (2000) las OAT debieran ser conformadas por propósitos responsables dirigidos a la consecución de valores éticos para la comunidad y los empleados. Esta claro que la actividad empresarial de una organización afecta a muchos grupos constituyentes o de referencia (proveedores, accionistas, clientes, consumidores, comunidad general, entre otros.), ya que esta vinculada a todos los grupos mencionados y con el mundo, por tanto desde el mismo momento en el cual se debilitan o rompen esos vínculos se promueven los desequilibrios éticos. Es en este momento cuando éste marco de relaciones se transplanta al territorio parti- 
Ética empresarial y el desempeño laboral en Organizaciones de Alta Tecnología (OAT) Ruiz C., Jocelyne K.; Silva V., Neif G. y Vanga A., María G.

cular de las OAT, se tiene que admitir el carácter diversificado y complejo de las relaciones que se imponen.

En el mismo orden de ideas es preciso abordar la relación que puede existir entre la ética y la cultura de la organización. En este sentido, con base en lo expuesto por Siliceo (1999), la cultura, entendida como el peculiar modo de la vida humana que cada grupo social se da, constituye la manera de entender el mundo y las formas de organización social dentro de las cuales todo grupo humano vive. Dicho de otra manera es un patrón de significados transmitidos a lo largo de la historia en símbolos, por medio de los cuales los hombres comunican, perpetúan y desarrollan su conocimiento y sus actividades en relación con la vida.

Así mismo, según Silva (2008) el ser humano y la cultura son realidades inseparables, ya que ésta última tiene como esencia unir, integrar, valorar, cultivar, en una palabra generar vida; funciones que no pueden existir sin la presencia de uno o del otro y así como los grupos humanos se suelen dividir, por razones de su propia organización interna, en subgrupos (por criterio de sexo, edad, ocupación, entre otros), la cultura, entendida como un árbol, tiene diversas ramificaciones a modo de subculturas que responden a las necesidades de organización y de significaciones específicas que cada grupo tiene.

Esta ramificación inevitable en donde se experimenta la micro cultura de una sociedad, es donde se sitúa la cultura organizacional, como contribución de la antropología aplicada y como instrumento en el ejercicio de la observación, análisis y desarrollo de las relaciones sociales que tienen lugar en una empresa como medio para lograr sus objetivos.

En referencia a lo expuesto, cabe decir que así como las culturas tribales tienen reglas y tabúes que dictan la forma en la que los miembros actuarán entre sí y con extraños, las organizaciones tienen culturas que dictan cómo deben comportarse sus miembros, en otras palabras, cada empresa posee sistemas o patrones de valores, símbolos, rituales, costumbres, creencias y prácticas que han evolucionado a lo largo del tiempo, y dichos valores compartidos determinan qué ven los empleados y como responden a su entorno, por lo que las empresas debieran adoptar una actitud responsable para dicha comunidad, ya que su comportamiento le repercute directa o indirectamente.

En este mismo orden de ideas, existen varios aspectos que juegan un papel importante en el buen desenvolvimiento de la actividad empresarial, uno de ellos es la responsabilidad, ya que se debe dirigir la empresa tomando en cuenta el impacto de su actuación en estos grupos, respetando sus derechos e intereses legítimos.

Es importante aclarar que en las OAT, en especial la honestidad, requiere de la rectitud y sinceridad con la tecnología manejada y con la información demandada por la comunidad, es decir, se requiere evitar el engaño y la desinformación.

Por otra parte, según la naturaleza de las OAT la confianza debe emerger como otro factor importante en las relaciones intra-organización, ya que se necesita del reconocimiento de compromisos implícitos en las promesas laborales y personales. En este sentido, la competencia leal y la consecución por la calidad 
real forman parte de la confianza, ya que su quebrantamiento perjudica en forma directa a las personas.

Asimismo, también se debe centrar la atención en tratar de actuar justamente tanto en el otorgamiento de oportunidades al interior de la empresa como a aquellos que tienen relación directa o indirecta con las actividades de la organización. Así como también evitar someterse a actividades impropias, favoritismos basados en intereses personales o presentar conductas que afecten la integridad de los ejecutivos ya que todas estas acciones contribuyen al quebrantamiento del código ético estipulado en la empresa.

De lo anteriormente expuesto, se afirma que actuar con integridad supone un comportamiento leal frente a las obligaciones y tareas que deben emprender los integrantes de la organización, es decir, se debe actuar conforme a las convicciones a los requerimientos morales, aunque presuponga un costo.

El comportamiento basado en estos valores como lo son la confianza, la honestidad, la integridad, los principios, entre otros, beneficia a toda la sociedad, a la empresa y a la economía en la cual se encuentra inserta la OAT, ya que determinaría la calidad de funcionamiento de la misma al lograr que los integrantes se identifiquen con los objetivos de la organización y combinen las acciones para cumplir dichas metas con las virtudes morales de cada uno de ellos.

De la misma forma, es importante el sentido de orgullo que puedan tener los empleados en su productividad y actitud general, cuando visualizan pertenecer a una organización que define los valores éticos como un estilo de vida.

\subsection{La ética y su influencia en las actitudes personales y organizacionales}

Una vez presentada la discusión sobre la ética empresarial y su relación con los valores y principios es necesario abordar la ética y su influencia en las actitudes personales y organizacionales.

A este respecto, el tener conciencia de los fines que se persiguen y habituarse a obrar con relación a ellos, es la clave de una ética de las personas y muy especialmente de las empresas. Asimismo, los modos de actuar ya asumidos, que disponen a obrar en el sentido deseado y que se han ido incorporando en los caracteres de las personas por repetición de actos, es a lo que tradicionalmente le llaman hábitos, los cuales cuando están bien orientados reciben el nombre de virtudes y cuando no se predisponen a alcanzar la meta, se denominan vicios.

Por otra parte, la ética, en un primer sentido, es un tipo de saber práctico, preocupado por averiguar cual debe ser el fin de las acciones del hombre; asimismo, para poder decidir sobre aspectos tales como: cuáles hábitos se deben asumir, cómo ordenar las metas intermedias, cuáles son los valores por los que se deben orientar y cuál modo de ser o carácter se debe incorporar. Todo esto con el objeto de obrar con prudencia, es decir, se refiere a tomar las decisiones acertadas teniendo como base la libertad de escoger un camino u otro, todo lo cual debe ir estrechamente ligado a la responsabilidad, que significa responder por lo que se ha elegido.

En otro orden de ideas, al hablar de actitudes se suele pensar en las personas, que pueden ser percibidas tanto por 
Ética empresarial y el desempeño laboral en Organizaciones de Alta Tecnología (OAT) Ruiz C., Jocelyne K.; Silva V., Neif G. y Vanga A., María G.

sus miembros internos como por aquellos ajenos a la organización. Si se piensa en la forma cómo deberían actuar las empresas, se debería tener en cuenta que desde el punto de vista de las personas, el saber ético orienta su actuación a fin de permitirles ser felices $u$ obtener un bienestar, el logro de la perfección y de la autorrealización.

Sin embargo, en lo que se refiere a las OAT, no tiene sentido creer que el fin de este tipo de organizaciones sea la felicidad, ya que felices son las personas, no las organizaciones, las cuales tienen otro tipo de metas. En este sentido, resulta más importante saber cuáles son las metas de la empresa, a fin de que sus miembros se esfuercen por alcanzarlas, siempre que sea retribuido este esfuerzo.

Según Leer (2000), las metas o fines en el entorno empresarial se fijan a través del diseño de un conjunto de reglamentos y normas; es por ello que el sentido de las actividades viene de los fines de la organización y las reglas sólo pueden fijarse teniendo en cuenta los fines, los cuales sin duda alguna son de carácter social.

Asimismo, es importante agregar que dichas reglas que se establecen para conducirse en la organización, no siempre se cumplen solo porque los integrantes deciden alcanzar el objetivo de la organización. Esto no es tarea sencilla, ya que el comportamiento ético de un empleado depende tanto de sus valores como del clima ético de la organización.

En tal sentido, puede ocurrir que el estimulo oriente a la gente a hacer cosas malas cuando el sistema de recompensas de su organización refuerza positivamente el comportamiento equivocado. Sin importar si lo que dice la administración es rele- vante, la gente de las organizaciones prestan atención a la forma en la que se manejan las verdaderas recompensas.

Por lo anteriormente expuesto se refuerza el concepto que el líder debe ocuparse de que sus empleados se comporten de acuerdo a las reglas de la organización, así como de generar un ambiente en el cual puedan actuar correctamente.

\subsection{Importancia de la ética en una empresa}

Hay asuntos organizacionales en los que una decisión es condicionada principalmente por los criterios éticos, y ello ha impulsado las investigaciones y análisis acerca de si es posible formular normas universales de conducta que permitan determinar cuando una decisión es mejor o peor que otras posibles soluciones. Más que una moda, la ética es en la actividad empresarial, para cualquier organización, una necesidad, una exigencia que se hace más apremiante conforme crece la complejidad del tejido social.

El tema de la ética en la mayoría de las empresas en la actualidad, más que un asunto de principios igualmente es de naturaleza estratégica, porque se vincula con la sobrevivencia de las mismas, y con la convivencia de las personas que trabajan en ellas.

Asimismo, el fin de las organizaciones guarda un fin social, porque toda organización se crea para proporcionar unos bienes o servicios, en virtud de los cuales queda legitimada su existencia ante la sociedad, y éste es un punto central en la elaboración de un código ético. 
Afirma Cortina (1998) que las organizaciones han de proporcionar unos bienes a la sociedad para ser aceptados por ella; lógicamente, en el caso de no producirlos, la sociedad tiene derecho a reclamarlos, y por último deslegitimarlas. Estos bienes se obtienen desarrollando determinadas actividades cooperativas. $Y$ aquí conviene recordar la distinción entre los bienes internos a una actividad cooperativa y los que son externos a ella.

Refiere Cortina (1998), que para diseñar una ética de las organizaciones sería necesario recorrer los siguientes pasos: determinar claramente el fin específico, el bien interno a la actividad que le corresponde y por el que cobra su legitimidad social; luego, averiguar cuáles son los medios adecuados para producir ese bien y qué valores es preciso incorporar para alcanzarlo; seguidamente, indagar qué hábitos han de ir adquiriendo la organización en su conjunto y los miembros que la componen para incorporar esos valores e ir forjándose en un carácter que les permita deliberar y tomar decisiones acertadas en relación con la meta.

Asimismo, es necesario discernir qué relación debe existir con las distintas actividades y organizaciones, así como también entre los bienes internos y externos a ellas; y por último, determinar cuáles son los valores de la moral cívica de la sociedad en la que se inscribe; después es necesario saber qué derechos reconoce esa sociedad a las personas. Es decir, cuál es la conciencia moral alcanzada por la sociedad.

Además se plantea cómo la distinción entre la racionalidad comunicativa y estratégica será de gran interés a la hora de construir una ética de la empresa; por- que tradicionalmente se suele entender a la empresa como regida por una racionalidad estratégica, dirigida a obtener el máximo beneficio, mientras que el momento moral es el de racionalidad comunicativa, apareciendo entonces, empresa y ética como aparentemente incompatibles.

Sin embargo, para Cortina (1998), cualquier ética aplicada (también empresarial) debe recurrir a estos dos tipos de racionalidad, porque ha de contar a la vez con estrategias y a su vez con una comunicación que le permita interactuar con interlocutores validos (clientes externos e internos).

\section{Desempeño laboral}

Para las organizaciones, el desempeño laboral no ha sido una novedad debido a que el individuo ha mantenido desde hace tiempo una relación con su trabajo. De tal forma, el desempeño laboral se puede entender como el merito que puede mejorar tanto a las organizaciones como al personal que las conforman.

Según Stoner (1994) el desempeño laboral "es la manera como los miembros de la organización trabajan eficazmente, para alcanzar metas comunes, sujeto a las reglas básicas establecidas con anterioridad".

Por otro lado, Chiavenato (1998) expone sobre desempeño laboral como "una sistemática apreciación del desempeño, del potencial de desarrollo del individuo en el cargo, afirmando que toda evaluación es un proceso para estimular o juzgar el valor, la excelencia, las cualidades de alguna persona".

Según estas definiciones se puede afirmar que el desempeño es una apre- 
Ética empresarial y el desempeño laboral en Organizaciones de Alta Tecnología (OAT) Ruiz C., Jocelyne K.; Silva V., Neif G. y Vanga A., María G.

ciación por parte de los supervisores de una empresa de la manera como un empleado logra sus metas u objetivos, así mismo, el desempeño laboral permite visualizar el punto hasta el cual un empleado puede realizar una labor; aclarando que dicho desempeño no solo incluye la producción de unidades tangibles sino también las no tangibles como lo es el pensar en forma creativa, inventar un producto nuevo, resolver un conflicto entre otros o vender un bien o servicio.

Ahora bien, desempeño laboral, es cuando ocurre en un contexto laboral, rodeado y regulado por normas, restricciones, expectativas de otros, exigencias, incentivos y probablemente recursos o ayudas necesarias para asegurar la calidad desempeño-resultado.

Al respecto, Harbour (1999) afirma que en el desempeño laboral intervienen factores o condiciones relacionados dentro del llamado contexto del desempeño, y son en primer lugar las condiciones antecedentes las cuales constituyen una fuente de causalidad desde remota o mediata del desempeño, que actúan sobre factores llamados intervinientes conformados por 3 categorías: las características de las condiciones sociales (status, roles), culturales (valores, creencias y actitudes) y demográficas (edad, ocupación, nivel socio-económico, estado civil, salud y educación); las categorías de los factores de personalidad: incluyen un conjunto de tenencias, patrones de comportamiento y reacciones de la persona; y las categorías de las características del puesto de trabajo: constituyen circunstancias o hechos externos a las personas; sus especificaciones y carac- terísticas inducen a ciertos patrones de acción en las personas que desempañan el cargo.

El mismo autor afirma, que las condiciones intervinientes las cuales son afectadas de algún modo por las condiciones antecedentes y sus categorías son: el conocimiento, el cual a su vez esta conformado por el cúmulo de conceptos, datos y hechos asimilados y estructurados por la persona a lo largo de su vida (en el caso del desempeño laboral, son los conocimientos que se adquieren mediante la experiencia y el aprendizaje, derivados del proceso de socialización y al mismo tiempo, influye el desempeño laboral de los individuos); las habilidades que representan un conjunto de operaciones intelectuales y motoras, que explican en gran parte el grado de desarrollo que una persona ha logrado de sus actividades asociadas al desempeño laboral; y la motivación en la cual se engloban factores y hechos que reciben la influencia de las condiciones (antecedentes sociales, culturales y demográficas) y pueden condicionar el desempeño laboral.

Apoyando lo anteriormente expuesto, vale la pena discernir sobre un término relacionado con el tema en cuestión como lo es la administración del desempeño, lo cual busca lograr los resultados esperados en la administración del recurso humano.

\subsection{Administración del desempeño}

La administración del desempeño es un enfoque sistemático aplicado a la administración de personal del día a día en el ambiente de trabajo, orientado a evaluar los resultados esperados en la 
ejecución de un proceso; utilizando el "acompañamiento" como recurso principal para optimizar los resultados. La misma posee un enfoque sistémico cuando se integran las acciones administrativas para disminuir individual y colectivamente los desempeños no deseados e incrementar los deseados.

También pretende dejar claros los resultados esperados en términos de calidad, costo y oportunidad, individual y grupal, para continuar con un proceso de seguimiento, la cual propende por prevenir un buen desempeño al acompañar al empleado y plantearle mejoras en los procesos que ejecuta, o en los resultados que debe alcanzar. Para lograrlo, es necesario hacer acuerdos dejando constancia de ellos, cada vez que su buen criterio administrativo se lo insinúe, o el resultado del empleado lo requiera, no cada tres, seis o doce meses como se hacía. Asimismo, la Administración del desempeño, insinúa medidores (costo-calidad-oportunidad), puesto que si no los hay, no será fácil cuantificar el desempeño. Si no los tiene, tendrá entonces que corregir y no prevenir, obteniendo resultados ineficientes. La medición reduce el sentimentalismo y aumenta la solución constructiva de los problemas.

Por otra parte, debe generar un ambiente en el que el empleado experimente ayuda para mejorar su desempeño al ejecutar un proceso y obtener un mejor resultado. No debe convertirse en una herramienta más para calificarlo y castigarlo, si el resultado es malo, será útil en el caso extremo en que deba tomar una acción drástica. En ésta, se puede justificar la decisión en los datos cuantificados y conservados como historia de los resultados alcanzados en acuerdos y re- visiones anteriores con el empleado. En este orden de ideas, se requiere de un buen equipo de trabajo que esté en condiciones de proyectar acciones cualitativas, para lograr la misión y visión de las instituciones.

Para tal efecto, se mencionan 5 principios que ayudaran a lograr dichos objetivos: la Planificación del Desempeño, donde establece lo que se espera de los trabajadores; el Desarrollo del Desempeño, aspecto en el cual se proveen las técnicas y los métodos apropiados para ayudar a los trabajadores; asimismo, la Revisión donde se efectúa una supervisión constante del desarrollo de los subordinados, el Monitoreo, donde juega un papel importante el gerente, pues le corresponde tener bien motivados al personal, para que ellos mejoren su desempeño; y finalmente, la Evaluación del Desempeño, es en este aspecto donde se observa y se reconoce si el docente se desenvuelve en forma efectiva, y dada su importancia al efectuar esta evaluación, se deben considerar ciertos aspectos como: centrar su acción en las tareas que corresponden, ya que esta se convierte en una estrategia de perfeccionamiento que permite ejercitar actividades en reflexión, cooperación y participación.

De lo anterior se deduce, que el desempeño laboral depende de las dinámicas de las relaciones que se establecen entre el gerente y los subordinados.

\subsection{Evaluación del desempeño laboral}

La evaluación del desempeño es una política de la dirección de personal, cuyo fin es detectar problemas en el tra- 
Ética empresarial y el desempeño laboral en Organizaciones de Alta Tecnología (OAT) Ruiz C., Jocelyne K.; Silva V., Neif G. y Vanga A., María G.

bajo para mejorar la eficiencia en la organización. En este sentido, Aragón (2004), afirma que la evaluación del desempeño es una práctica integrada dentro de una filosofía de comunicación entre superior y subordinado, con la que se revisan los aspectos claves del trabajo.

De acuerdo a estas consideraciones, uno de los objetivos de la evaluación del desempeño es la valoración del empleado en el puesto de trabajo para conocer su actuación y su factible potencial de desarrollo. Asimismo, este proceso, estimará los valores, excelencia, cualidades y estatus de las personas.

En el mismo marco de ideas, Puchol (2005) afirma que la evaluación del desempeño es un procedimiento continuo de expresión de juicios acerca del personal de una empresa, en relación con su trabajo habitual, que pretende sustituir a los juicios ocasionales y formulados de acuerdo con los más variados criterios.

Por consiguiente, estos autores, convergen en que la evaluación de la labor es el proceso de medir la actuación en el trabajo de los subordinados para conocer su desempeño, y cual es su potencial de desarrollo en la organización.

En resumidas cuentas, la evaluación del desempeño es una técnica indispensable en la administración de personal que contribuye al autodesarrollo del empleado y a su vez, puede detectar algunas situaciones relevantes en el trabajo, tales como: problemas de supervisión de personal, integración del personal a la empresa, deficiencia de motivación, y según el problema que se detecte, puede colaborar con la implantación de políticas adecuadas a la realidad de la institución.
Según Chiavenato (1998), la evaluación del desempeño va estrictamente vinculada a los incentivos y las motivaciones personales, las correcciones de los problemas confrontados y la acción gerencial. Cuando se siguen estos pasos, la persona se siente participe de los objetivos de la organización, mejoran las relaciones y emplean los medios necesarios para mejorar el desempeño actual.

En línea con lo discutido hasta ahora, se puede decir que el proceso de evaluación de desempeño, constituye el paso en el cual se estima el rendimiento global del empleado. La mayor parte de los empleados procuran obtener retroalimentación sobre la manera cómo se cumplen sus actividades y las personas que tienen a su cargo la dirección de otros empleados deben evaluar el desempeño individual para decidir las acciones que deben tomar.

Al respecto Chiavenato (1998), indica que las evaluaciones informales, basadas en el trabajo diario, son necesarias pero insuficientes. Contando con un sistema formal y sistemático de retroalimentación, el departamento de personal puede identificar a los empleados que cumplen o exceden lo esperado y a los que no lo hacen. Asimismo, ayuda a evaluar los procedimientos de reclutamiento, selección y orientación. Incluso las decisiones sobre promociones internas, compensaciones y otras más del área del departamento de personal dependen de la información sistemática y bien documentada disponible sobre el empleado.

Por otro lado, además de mejorar el desempeño, muchas compañías utilizan esta información para determinar las compensaciones que otorgan. Un buen 
sistema de evaluación puede también identificar problemas en el sistema de información sobre recursos humanos. Las personas que se desempeñan de manera insuficiente pueden poner en evidencia procesos equivocados de selección, orientación y capacitación, o puede indicar que el diseño del puesto o los desafíos externos no han sido considerados en todas sus facetas.

Una organización no puede adoptar cualquier sistema de evaluación del desempeño. El sistema debe ser válido y confiable, efectivo y aceptado. El enfoque debe identificar los elementos relacionados con el desempeño, medirlos y proporcionar retroalimentación a los empleados y al departamento de personal.

Por norma general, el departamento de recursos humanos desarrolla evaluaciones del desempeño para los empleados de todos los departamentos. Esta centralización obedece a la necesidad de dar uniformidad al procedimiento. Aunque el departamento de personal puede desarrollar enfoques diferentes para ejecutivos de alto nivel, profesionales, gerentes, supervisores, empleados y obreros, necesitan uniformidad dentro de cada categoría para obtener resultados utilizables. Aunque es el departamento de personal el que diseña el sistema de evaluación, en pocas ocasiones lleva a cabo la evaluación misma, que en la mayoría de los casos es tarea del supervisor del empleado.

La evaluación del desempeño laboral evidencia algunas ventajas, lo cual resulta importante abordar. En este sentido, se enumeran algunas que ha podido ser verificadas: la mejora del desempeño mediante la retroalimentación, las políticas de compensación que ayudan a determinar quiénes merecen recibir aumentos, las decisiones de ubicación que generan promociones, transferencias y separaciones basadas en el desempeño anterior o en el previsto, las necesidades de capacitación y desarrollo, en las cuales el desempeño insuficiente puede indicar la necesidad de volver a capacitar, a un potencial no aprovechado.

En este sentido, la evaluación del desempeño laboral es de suma importancia para las organizaciones, así como también para la nación en general, ya que esto podría incrementar el rendimiento individual en cada puesto de trabajo, creciendo así la formación integral del ser humano como ente social significativo para el trabajo; y al mismo tiempo, abriendo la posibilidad de aumentar la productividad y la estabilidad económica de la sociedad en la que convive.

\section{Relación entre ética empresarial y desempeño laboral}

La meta de la actividad empresarial es la satisfacción de necesidades humanas a través de la inversión de un capital, que es parte esencial el capital humano, es decir, los recursos humanos o dicho de otra forma, las capacidades de cuantos cooperan en la empresa.

Por tanto, el fin interno de las empresas consiste en lograr satisfacer esas necesidades $\mathrm{y}$, de forma inseparable, en desarrollar al máximo las capacidades de sus colaboradores para lograr un desempeño laboral deseable, metas ambas que no podrá alcanzar si no es promocionando valores desde el modo especifico en 
Ética empresarial y el desempeño laboral en Organizaciones de Alta Tecnología (OAT) Ruiz C., Jocelyne K.; Silva V., Neif G. y Vanga A., María G.

que la empresa puede y debe hacerlo. Asimismo, al hablar de satisfacer necesidades humanas se estaría hablando de servicio, donde el objetivo primordial es lograr la satisfacción del cliente y superar sus expectativas, lo que se logra ofreciendo un excelente servicio con los estándares éticos que posee la empresa y los valores del capital humano, logrando así el desempeño laboral apropiado.

De allí que se promueve una correlación entre ética y servicio, o lo que es similar ética empresarial y desempeño laboral. El servicio es un atributo de esencia ética, y la ética es filosofía traducida en servicio. En definitiva, el carácter activo de una empresa procede de la capacidad de dar, más que de la posibilidad de recibir. Esto significa que su potencialidad competitiva y su margen cooperativo proceden de la ética, de su atmósfera organizacional y del servicio que pauta su desempeño. La correspondencia entre ética y servicio viene acompañada por el rechazo de sus desviaciones y sesgos: servicio no es servilismo, como ética no es ascetismo.

Todos pueden servir así como todos deben asumir una conducta ética sin traspasar los umbrales de suprema dignidad y sin caer en los extremos de sus caricaturas. El servicio y la ética de manera semejante a lo que ocurre con el conocimiento crecen mientras más se traducen en ejecuciones concretas. También se produce una semejanza entre ambos aspectos, en cuanto a que ellos generan más dividendos y acrecientan más la imagen de las organizaciones que la que procede por la venta de productos tangibles.

Es en este sentido, en el que la ética de la empresa tiene por valores irre- nunciables la calidad en los productos y la gestión, la honradez en el servicio, el mutuo respeto en las relaciones internas y externas a la empresa, la cooperación por la que conjuntamente se aspira a la calidad y la solidaridad al alza. Los valores anteriormente expuestos consisten en explotar al máximo las propias capacidades de modo que el conjunto de personas pueda beneficiarse de ellas, la creatividad, la iniciativa, el espíritu de riesgo; valores que definitivamente lograran que los colaboradores de una empresa obtengan un excelente desempeño laboral movidos en conjunto por un interés común, el mismo que persigue la organización.

\section{Discusión de los resultados}

Esta se realiza con base en los resultados obtenidos en los diferentes indicadores de cada dimensión en el marco de cada variable de investigación, a saber la Ética Empresarial y El Desempeño Laboral; y en consideración de la población del grupo de gerentes de diferentes Organizaciones de Alta Tecnología.

A partir de la aplicación de los instrumentos de recolección de información diseñados en correspondencia con el esquema de operacionalización de las variables se presentan los resultados de la data obtenida en el ámbito de la población de gerentes de OAT estudiada. Tal presentación se hace mediante la tabulación en cuadros que muestran tanto las cantidades asignadas a cada alternativa de respuesta, las cuales indican la frecuencia absoluta, como sus porcentajes.

Así mismo, el análisis de resultados se realiza bajo el marco de las variables, dimensiones e indicadores de esta 
investigación, mediante la utilización de referenciales de hallazgos similares en los antecedentes de la investigación y/o sobre la base de los planteamientos establecidos en las bases teóricas.

Enmarcados en la variable ética empresarial y dentro de la dimensión Valores, cuyos indicadores son: puntualidad, confianza, respeto, honestidad y responsabilidad, resulta de interés indicar que la población estudiada manifiesta de manera significativa, que los elementos de valores se ajustan al cumplimiento del código de ética de las empresas y lo califican como un factor Muy Importante en el entorno donde cada uno de ellos se desenvuelve (ver Tabla 1). Como tal, esta situación proyecta una adopción relativamente alta del código de ética de las empresas, lo cual sienta las bases para que los directivos encargados de llevar el control de las organizaciones continúen perfeccionando sus acciones, su capacidad de autogobernarse e inclusive el uso de su libertad, lo cual puede traducirse en la capacidad de superar nuevos retos con y para el cliente.

Sin embargo, para llevar a cabo nuevos proyectos aún más conscientemente apegados al código de ética, las empresas deberán prestar atención a solventar las debilidades que se identificaron en cuanto a algunos factores éticos, como lo son: la confianza y la puntualidad que aún cuando reflejaron niveles altos, también tienen una marcada tendencia en contraposición de algunos otros encuestados. Estos resultados tienen coincidencia con algunos de los trabajos consultados, pero más firmemente se encuentra en la revisión teórica realizada
Tabla 1

Variable: Ética empresarial Dimensión: Valores

\begin{tabular}{lcccc}
\hline Valores & Rangos & FA & fa & $\%$ \\
\hline Muy & & & & \\
importante & $\geq 69-92$ & 54 & $54 / 54$ & $100 \%$ \\
Importante & $\geq 46-<69$ & 0 & $0 / 54$ & $0 \%$ \\
Poco & & & & \\
importante & $23-<46$ & 0 & $0 / 54$ & $0 \%$ \\
Totales & & 54 & 1 & $100 \%$ \\
\hline
\end{tabular}

Fuente: Ruiz (2007).

los aportes de Pérez (1998) quien afirma que los valores éticos son aquellas realidades cuya posesión perfecciona al ser humano en lo más profundo de su ser: perfecciona su capacidad de autogobierno y libertad.

Por otra parte, según Siliceo (1997) menciona que los valores deben ser asumidos ampliamente por el personal que labora en las empresas a fin de garantizar en alguna medida altos rendimientos en la capacidad de servicio y de respuesta; por lo que es recomendable elevar los niveles de cumplimiento de éstos y todos los factores éticos ya asumidos por el grupo de empleados de los gerentes encuestados en este estudio.

En cuanto a la dimensión Creencias, la cual se analiza partiendo de las individuales y las creencias grupales, los porcentajes se manifiestan un poco más bajos en comparación con la dimensión anterior (ver Tabla 2). En efecto, el $74,07 \%$ de los encuestados reportan la identificación de dicho factor como muy arraigado dentro de las labores de sus empleados, lo que proyecta un saldo de cumplimiento favorable en las organizaciones, ya que evidencia la alta capaci- 
Ética empresarial y el desempeño laboral en Organizaciones de Alta Tecnología (OAT) Ruiz C., Jocelyne K.; Silva V., Neif G. y Vanga A., María G.

Tabla 2

Variable: Ética empresarial Dimensión: Creencias

\begin{tabular}{lcccc}
\hline Creencias & Rangos & FA & fa & $\%$ \\
\hline Muy & & & & \\
arraigada & $\geq 24-32$ & 40 & $40 / 54$ & $74,07 \%$ \\
Arraigada & $\geq 16-<24$ & 14 & $54 / 54$ & $25,93 \%$ \\
Poco & & & & \\
arraigada & $8-<16$ & 0 & $0 / 54$ & $0 \%$ \\
Totales & & 54 & 1 & $100 \%$ \\
\hline
\end{tabular}

Fuente: Ruiz (2007).

dad de adaptación de los empleados a los requerimientos éticos que exigen las empresas.

Los resultados para los indicadores de esta dimensión coinciden parcialmente con los resultados de García y Dolan (1997), quien ha concluido que la mayor afinidad en las creencias individuales y grupales que pueda darse en la organización, guarda una relación directa con la capacidad que han tenido los empleados de la misma en absorber y adaptarse a los posibles cambios que pueda insertar un código de ética en la empresa.

Por otra parte se denota que en el caso particular de la dimensión Principios, los resultados tienden a ser muy favorables según algunos encuestados, evidenciando porcentajes relativamente altos en cuanto a su relevancia, un promedio del $42,59 \%$ refleja como Importante los principios que pueden tener los integrantes en OAT (ver Tabla 3). Cabe señalar que en este caso en particular donde se discuten los principios tanto organizacionales como individuales, otro grupo de encuestados (el 5,56\%) no muestra resultados muy halagadores.
Tabla 3

Variable: Ética empresarial Dimensión: Principios

\begin{tabular}{lcccc}
\hline Principios & Rangos & FA & fa & $\%$ \\
\hline Muy & & & & \\
importante & $\geq 18-24$ & 28 & $28 / 54$ & $51,85 \%$ \\
Importante & $\geq 12-<18$ & 26 & $54 / 54$ & $42,59 \%$ \\
Poco & & & & \\
importante & $6-<12$ & 0 & $0 / 54$ & $5,56 \%$ \\
Totales & & 54 & 1 & $100 \%$ \\
\hline
\end{tabular}

Fuente: Ruiz (2007).

Los hallazgos para los factores éticos de esta dimensión coinciden con los resultados reportados por Guedez (2001), quien señala que la presencia de los principios éticos, puede incrementar la eficiencia del servicio sobre las necesidades del entorno productivo de la empresa. Por su parte, los elementos que reportan una menor utilización guardan coincidencia con los hallazgos de García y Dolan (1997), quienes han indicado que la ausencia de estos factores limitan en cierta medida la gestión de los procesos internos de la empresa, y por consiguiente, el desempeño laboral del individuo.

Ciertamente, a la luz de los resultados reportados se observa que la población de directivos de empresas de alta tecnología encuestada manifiesta que en su mayoría los factores éticos que conforman los conjuntos de principios individuales y organizacionales reportan marcada tendencia hacia el mayor cumplimiento del código de ética de cada una de las empresas a las que pertenecen. Cabe decir que, en posición contraria, algunos otros no muestran resultados alentadores. 
En el mismo orden de ideas, y según las teorías de García y Dolan (1997) acerca de ética empresarial, esta última situación reduce las posibilidades de consolidar un alto nivel de desempeño laboral; el mismo afirma que muchos de los principios individuales influyen en el desempeño del grupo que integra la organización. Esta posición anterior conduce a sugerir la tarea de diseñar e implementar los mecanismos necesarios a fin de consolidar los principios organizacionales sobre los individuales para mejorar el rendimiento de los procesos internos de cada organización.

Asimismo, analizando los datos obtenidos en cuanto a la dimensión Cultura, los hallazgos para los factores éticos sobre la misma muestran los esfuerzos de las organizaciones en materia de cultura (ver Tabla 4), ya que un $94,44 \%$ de los encuestados evidencian estar de acuerdo en calificar como muy Importante la existencia de factores culturales, el resto apoya en un 5,56\% calificando como Importante la presencia de dichos elementos en la empresa, lo cual es favorable y demuestra en alguna medida la identificación y el interés del grupo de gerentes de OAT por promover el proceso de adopción de los elementos que conforman la cultura de la empresa para nutrir los conocimientos y aportar más en sus labores diarias.

Dichos resultados corresponden con las opiniones que ofrece Siliceo (1999), quien señala que la cultura es un conjunto de elementos fundamentales, compartidos grupalmente y sedimentados a lo largo de la vida de la empresa a la cual se identifican, estos son transmitidos a sus miembros y ayudan en la resolución de problemas.
Tabla 4 Variable: Ética empresarial Dimensión: Cultura

\begin{tabular}{lcccc}
\hline Cultura & Rangos & FA & fa & $\%$ \\
\hline Muy & & & & \\
importante & $\geq 24-32$ & 51 & $51 / 54$ & $94,44 \%$ \\
Importante & $\geq 16-<24$ & 3 & $54 / 54$ & $5,56 \%$ \\
Poco & & & & \\
importante & $8-<16$ & 0 & $0 / 54$ & $0 \%$ \\
Totales & & 54 & 1 & $100 \%$ \\
\hline
\end{tabular}

Fuente: Ruiz (2007).

Por otro lado, refiriendo el análisis hacia la dimensión Moral, para lo cual se tomó en cuenta en forma individual y organizacional resulta interesante indicar que el grupo de empleados manifiesta que la moral es un elemento importante para lograr una ética deseada dentro de las empresas (ver Tabla 5). Así que, esta situación proyecta una adopción relativamente alta del código de ética de la empresa, lo cual muestra una presencia de factores morales en los empleados de las empresas, y esto, a su vez estaría garantizando en alguna medida la realización de actividades claras y limpias para con el cliente.

Asimismo, según la revisión teórica realizada, Etkin (1993) afirma que la moral debe ser asumida por quienes conforman el grupo de trabajo a fin de garantizar en alguna medida altos rendimientos en la calidad de servicio; por lo que es recomendable elevar aún más el nivel de cumplimiento de éstos y todos los factores éticos ya asumidos por los integrantes de OAT.

En otro orden de ideas se analiza la dimensión Responsabilidad, donde se observa que los integrantes del grupo de 
Ética empresarial y el desempeño laboral en Organizaciones de Alta Tecnología (OAT) Ruiz C., Jocelyne K.; Silva V., Neif G. y Vanga A., María G.

Tabla 5

Variable: Ética empresarial Dimensión: Moral

\begin{tabular}{lcccc}
\hline Moral & Rangos & FA & fa & $\%$ \\
\hline Muy & & & & \\
importante & $\geq 18-24$ & 21 & $21 / 54$ & $38,88 \%$ \\
Importante & $\geq 12-<18$ & 30 & $51 / 54$ & $55,56 \%$ \\
Poco & & & & \\
importante & $6-<12$ & 3 & $54 / 54$ & $5,56 \%$ \\
Totales & & 54 & 1 & $100 \%$ \\
\hline
\end{tabular}

Fuente: Ruiz (2007).

directivos, manifiestan de manera significativa que existe un elevado cumplimiento de la responsabilidad por parte de sus empleados, basado en el compromiso que se le debe tanto a los proveedores, clientes externos, clientes internos y a la sociedad, indicadores éstos que evidencian en el ejercicio de las funciones de los empleados, el apego al código de conductas éticas que posee cada organización con sus trabajadores (ver Tabla 6).

Por tanto, el efecto que pueden tener estos factores éticos sobre el desempeño de las labores diarias de los integrantes de una organización, tiende en su mayoría a ser favorable, ya que todos los indicadores sin excepción evidencian porcentajes por encima del $60 \%$, lo cual induce un nivel de aplicación del código de ética importante.

Asimismo, se observa cómo es adoptada la ética en las empresas por parte de la población encuestada, los resultados muestran, que un $100 \%$ de los gerentes opina que se cumple el código de conductas éticas que posee sus empresas para guiar y justificar las acciones y decisiones en la organización, aplicando los valores, principios, creencias de
Tabla 6 Variable: Ética empresarial Dimensión: Responsabilidad

\begin{tabular}{lllcc}
\hline Responsabilidad & Rangos & FA & fa & $\%$ \\
\hline $\begin{array}{l}\text { Elevado } \\
\text { cumplimiento }\end{array}$ & $\geq 51-68$ & 54 & $54 / 54$ & $100 \%$ \\
$\begin{array}{l}\text { Mediano } \\
\text { cumplimiento }\end{array}$ & $\geq 34-<51$ & 0 & $0 / 54$ & $0 \%$ \\
$\begin{array}{l}\text { Bajo } \\
\text { cumplimiento }\end{array}$ & $17-<34$ & 0 & $0 / 54$ & $0 \%$ \\
Totales & & 54 & 1 & $100 \%$ \\
\hline Fuente: Ruiz (2007). & & &
\end{tabular}

cada uno de ellos y cumpliendo con los compromisos que se tienen con los clientes y la sociedad (ver Tabla 7).

Por otra parte, enmarcados en la Variable Desempeño laboral y refiriendo la Dimensión: Aportes o resultados, se observa a la luz de los resultados reportados, que los directivos evidencian un puntaje favorable con respecto a los indicadores asociados a esta dimensión (ver Tabla 8). El (100\%) se concentra en aspectos positivos, lo que indica que parece existir una clara conciencia acerca de cómo se realizan las actividades de los empleados, y que induce a su vez un alto nivel de desempeño laboral.

Como tal, estos resultados vienen a guardar coincidencia con los señalamientos de Werther (2001), quien plantea que uno de los factores de éxito a través del cual los integrantes de un grupo de trabajo comienzan a dominar el rendimiento del grupo es a través de las consecuencias finales de cada labor. Agrega además, que estas consecuencias sirven como alternativa organizacional para facilitar la implementación de estrategias adecuadas que se traduzcan en mejoras del desempeño organizacional. 
Tabla 7

Variable: Ética empresarial

\begin{tabular}{|c|c|c|c|c|}
\hline $\begin{array}{l}\text { Variable Ética } \\
\text { Empresarial }\end{array}$ & Rangos & FA & fa & $\%$ \\
\hline $\begin{array}{l}\text { Elevado } \\
\text { cumplimiento }\end{array}$ & $\geq 204-272$ & 254 & $54 / 54$ & $100 \%$ \\
\hline $\begin{array}{l}\text { Mediano } \\
\text { cumplimiento }\end{array}$ & $\geq 136-204$ & 40 & $0 / 54$ & $0 \%$ \\
\hline $\begin{array}{l}\text { Bajo } \\
\text { cumplimiento }\end{array}$ & $68-<136$ & 0 & $0 / 54$ & $0 \%$ \\
\hline Totales & & 54 & 1 & $100 \%$ \\
\hline $\begin{array}{l}\text { Variable: } \\
\text { Dimensión }\end{array}$ & $\begin{array}{r}\text { Tabla } 8 \\
\text { Desempє } \\
\text { : Aportes }\end{array}$ & $\begin{array}{l}8 \\
\text { eño } \\
\text { s o r }\end{array}$ & $\begin{array}{l}\text { Labo } \\
\text { resulta }\end{array}$ & ados \\
\hline $\begin{array}{l}\text { Aport } \\
\text { Resul }\end{array}$ & Rangos & FA & fa & $\%$ \\
\hline $\begin{array}{l}\text { Bajo } \\
\text { Desempeño }\end{array}$ & $\geq 42-56$ & 54 & $54 / 54$ & $100 \%$ \\
\hline $\begin{array}{l}\text { Mediano } \\
\text { Desempeño }\end{array}$ & $\geq 28-<42$ & 0 & $0 / 54$ & $0 \%$ \\
\hline $\begin{array}{l}\text { Alto } \\
\text { Desempeño }\end{array}$ & $14-<28$ & 0 & $0 / 54$ & $0 \%$ \\
\hline Totales & & 54 & 1 & $100 \%$ \\
\hline
\end{tabular}

Asimismo, dentro de la dimensión: Competencias Técnicas, los resultados muestran que un $95 \%$ del grupo de directivos, manifiestan que sus subordinados poseen las competencias técnicas en un Alto Desempeño (ver Tabla 9).

En su gran mayoría los integrantes de OAT poseen un adecuado nivel de instrucción para el servicio que prestan y cada uno se preocupa por adquirir los conocimientos que están en la vanguardia tecnológica, además de existir un apoyo completamente inclinado hacia un interés global de alcanzar las metas trazadas.
Tabla 9

Variable: Desempeño Laboral Dimensión: Competencias Técnicas

\begin{tabular}{lcccc}
\hline $\begin{array}{l}\text { Competencias } \\
\text { Técnicas }\end{array}$ & Rangos & FA & fa & $\%$ \\
\hline $\begin{array}{l}\text { Alto } \\
\text { Desempeño }\end{array}$ & $\geq 45-60$ & 54 & $54 / 54$ & $100 \%$ \\
$\begin{array}{l}\text { Mediano } \\
\text { Desempeño }\end{array}$ & $\geq 30-<45$ & 0 & $0 / 54$ & $0 \%$ \\
Bajo & & & & \\
Desempeño & $15-<30$ & 0 & $0 / 54$ & $0 \%$ \\
Totales & & 54 & 1 & $100 \%$ \\
\hline
\end{tabular}

Fuente: Ruiz (2001).

De la misma forma se denotan los aspectos resaltantes en cuanto al desempeño de las Competencias Generales,siendo que el proceso de gerencia en organizaciones que prestan asistencia tecnológica debe centrar sus esfuerzos hacia la adopción de modalidades que favorezcan el servicio prestado a los clientes, y por consiguiente el buen desempeño que esto pueda representar para cada integrante de la organización; resulta reconfortante que los porcentajes alcanzados evidencien una tendencia alta del 100\% (ver Tabla 10).

El espectro de modalidades de servicio y las distintas conductas individuales para la proyección del grupo de empleados de los gerentes encuestados, se observan en cierta medida sobre una base sólida dado los resultados evidenciados en cada uno de los indicadores de esta dimensión como lo son: colaboración, carácter, apariencia y valores. Según los resultados de estudios empíricos reportados por Chiavenato (1998), se puede apreciar cierto nivel de coincidencia al señalar que todo esquema de traba- 
Ética empresarial y el desempeño laboral en Organizaciones de Alta Tecnología (OAT) Ruiz C., Jocelyne K.; Silva V., Neif G. y Vanga A., María G.

Tabla 10

\section{Variable: Desempeño Laboral Dimensión: Competencias Generales}

\begin{tabular}{lcccc}
\hline $\begin{array}{l}\text { Competencias } \\
\text { Generales }\end{array}$ & Rangos & FA & fa & $\%$ \\
\hline $\begin{array}{l}\text { Alto } \\
\text { Desempeño }\end{array}$ & $\geq 54-72$ & 54 & $54 / 54$ & $100 \%$ \\
Mediano & & & & \\
Desempeño & $\geq 36-<54$ & 0 & $0 / 54$ & $0 \%$ \\
$\begin{array}{l}\text { Bajo } \\
\text { Desempeño }\end{array}$ & $18-<36$ & 0 & $0 / 54$ & $0 \%$ \\
Totales & & 54 & 1 & $100 \%$ \\
\hline Fuente: Ruiz (2007). & & &
\end{tabular}

jo grupal tiende a hacerse más efectivo en la medida que logra implementar un mayor conjunto de modalidades que incrementen las posibilidades de cooperación entre los integrantes del grupo, y a su vez, favorece al mismo tiempo el mejoramiento del desempeño laboral.

Por último, analizando los resultados de la Dimensión: Mejoramiento Académico, y considerando los indicadores: adiestramiento y desarrollo, los mismos sugieren una orientación y visión clara por parte de la gerencia hacia la preparación efectiva de los integrantes de una organización, sobre todo en lo que respecta a la ausencia evidenciada en alguna medida en la organización de charlas y reuniones orientadas al mejoramiento de los conocimientos del empleado, y en la planificación de cursos de actualización, capacitación y desarrollo (ver Tabla 11).

De esta manera, las empresas deberían reorientar los esfuerzos internos a fin de reestructurar su visión gerencial acerca del servicio que se está prestando hacia los clientes, a fin de proporcionar al
Tabla 11

Variable: Desempeño Laboral Dimensión: Mejoramiento Académico

\begin{tabular}{lcccc}
\hline $\begin{array}{l}\text { Mejoramiento } \\
\text { Académico }\end{array}$ & Rangos & FA & fa & $\%$ \\
\hline $\begin{array}{l}\text { Alto } \\
\text { Desempeño }\end{array}$ & $\geq 12-16$ & 24 & $24 / 54$ & $44,44 \%$ \\
$\begin{array}{l}\text { Mediano } \\
\text { Desempeño }\end{array}$ & $\geq 8-<12$ & 30 & $30 / 54$ & $55,56 \%$ \\
$\begin{array}{l}\text { Bajo } \\
\text { Desempeño }\end{array}$ & $4-<8$ & 0 & $0 / 54$ & $0 \%$ \\
Totales & & & & \\
\hline Fuente: Ruiz (2007). & 54 & 1 & $100 \%$ \\
\hline
\end{tabular}

empleado, las oportunidades de realizar cursos de actualización y de participar con mayor frecuencia en charlas donde se compartan conocimientos entre los mismos integrantes de la organización, todo esto planificado y organizado de acuerdo a las necesidades individuales y grupales.

Esta sugerencia se apoya en los planteamientos señalados por Chiavenato (1998), quien plantea que en la medida que la organización desarrolle esfuerzos que convengan tanto para los empleados como para los clientes y por ende, para la misma compañía inclusive, en esa misma medida se tiende a viabilizar la posibilidad de incrementar de manera exponencial el rendimiento de los empleados y la calidad en el servicio prestado.

En este caso particular, los resultados sugieren una leve tendencia para viabilizar el fortalecimiento de las debilidades encontradas en esta dimensión, la planificación de cursos y reuniones donde la intención sea adquisición y actualización de conocimientos, así como su aplicación y empleo en los procesos em- 
presariales. De hecho, se incrementan las posibilidades de hacer sentir la capacidad tecnológica, el grupo en el entorno empresarial y, con ello la capacidad de respuesta que las empresas puedan incorporar al proceso de servicio a sus clientes.

En referencia al análisis de la variable desempeño laboral de los integrantes de la organización por parte del grupo de directivos encuestados, y tomando en cuenta todas sus dimensiones, se denota que ejercen un nivel de desempeño bastante alto, ya que en la mayoría de los casos brindan los resultados esperados por la empresa, el empleado se preocupa por ser cada vez más competitivo, tanto técnicamente como en general y logra mantenerse a la vanguardia asistiendo a cursos, eventos y congresos de actualización que le permitirán brindar un mejor servicio (ver Tabla 12).

Por último los resultados obtenidos en el cálculo de la relación entre ética empresarial y desempeño laboral señalan una proporción positiva muy débil, lo cual
Tabla 12 Variable: Desempeño Laboral

\begin{tabular}{|c|c|c|c|c|}
\hline $\begin{array}{l}\text { Variable } \\
\text { Desempeño } \\
\text { Laboral }\end{array}$ & Rangos & FA & fa & $\%$ \\
\hline $\begin{array}{l}\text { Alto } \\
\text { Desempeño }\end{array}$ & $\geq 153-204$ & 54 & $54 / 54$ & $100 \%$ \\
\hline $\begin{array}{l}\text { Mediano } \\
\text { Desempeño }\end{array}$ & $\geq 102-153$ & 0 & $0 / 54$ & $0 \%$ \\
\hline $\begin{array}{l}\text { Bajo } \\
\text { Desempeño }\end{array}$ & $51-<102$ & 0 & $0 / 54$ & $0 \%$ \\
\hline Totales & & 54 & 1 & $100 \%$ \\
\hline
\end{tabular}

Fuente: Ruiz (2007).

indica que a media que la variable ética empresarial crece una unidad, en cualquier ponderación, la variable desempeño laboral se ve afectada en la misma dirección en una muy poca y débil proporción (ver Tabla 13).

Por lo tanto, se puede señalar que los resultados de esta investigación, indican que en base a la metodología científica aplicada, la influencia entre las variables en estudio, no guardan una significante relación entre sí.

Tabla 13

Cálculo de la relación entre Ética Empresarial y Desempeño Laboral

\begin{tabular}{|l|c|c|c|c|c|}
\hline Variables & $\Sigma \mathrm{X} 2$ & $\Sigma Y 2$ & $\Sigma \mathrm{X}$ & $\Sigma \mathrm{Y}$ & $\mathrm{X} . \mathrm{Y}$ \\
\hline Sumatoria & 142969849 & 1543653 & 11957 & 9123 & 2023154 \\
\hline
\end{tabular}

$\begin{array}{ll}\text { Ética Empresarial }=X & N=54 \\ \text { Desempeño Laboral }=Y & X=11957 \\ Y=9123\end{array}$

Cálculo de la correlación por el medio de las calificaciones originales

$r t t=\frac{\frac{\sum X Y-\left(\sum X\right)\left(\sum Y\right)}{N}}{\sqrt{\left(\sum X^{2}-\sum X^{2} / N\right)\left(\sum Y^{2}-\sum Y^{2} / N\right)}}=r t t=0,000211601$ 
Ética empresarial y el desempeño laboral en Organizaciones de Alta Tecnología (OAT) Ruiz C., Jocelyne K.; Silva V., Neif G. y Vanga A., María G.

\section{Conclusiones}

Tomando como referencia el análisis y la discusión de los resultados de la investigación, se formula un grupo de conclusiones de acuerdo al objetivo planteado.

Al momento de identificar los componentes éticos de mayor importancia en el personal adscrito a cada uno de los gerentes de OAT encuestados, se evidenció una marcada tendencia hacia los altos niveles de importancia de la mayoría de los componentes éticos, por lo que en este caso se puede concluir que el personal en posición gerencial tiene una percepción favorable sobre su equipo de trabajo, en relación a los elementos éticos encontrados en el personal.

Asimismo, a través del análisis realizado al componente ético de Responsabilidad, se evidenciaron cifras unánimes hacia un Elevado Cumplimiento, lo cual refiere la existencia de un acentuado cumplimiento del código de ética en las empresas a las que pertenecen los gerentes encuestados en este estudio. Es posible afirmar que se comprobó en cierta medida la capacidad de respeto por las normas éticas de la organización.

Por otra parte, a pesar de la marcada tendencia hacia el Alto Desempeño evidenciada en las dimensiones que componen la variable Desempeño Laboral y el Mejoramiento Académico, es oportuno sugerir una reorientación de los esfuerzos internos a fin de proporcionar al empleado una mayor participación a cursos de capacitación, talleres o charlas donde se compartan conocimientos entre los mismos integrantes de la organización, todo esto planificado y organizado de acuerdo a las necesidades individuales y grupales.

En líneas generales, la correspondencia entre la ética empresarial y el desempeño laboral de una organización se pudo evidenciar a través del cálculo de la relación entre cada una de las variables, a partir de lo cual arrojo como resultado un $\mathrm{rtt}=0,0002$, lo cual se interpretó como una relación positiva muy débil entre las dos variables, lo que significa que mientras el empleado mejore el cumplimiento de la ética en sus labores, no necesariamente se evidenciará en él algún progreso del desempeño laboral.

Aunado a esto, se puede afirmar que el buen comportamiento ético que mantenga el empleado no necesariamente obtendrá beneficios sustanciales y visibles de inmediato, sino, a largo plazo, lo que significará para las organizaciones de OAT una garantía de supervivencia, donde las empresas más responsables son las que han tenido mejoras.

Asimismo, el compromiso con un comportamiento ético y la disposición para aplicar instrumentos que permitan medir su cumplimiento se hacen prioritarios, y no sólo para esperar reducir los márgenes de error en las tomas de decisiones y formas de actuación, sino, para evitar manchas irreversibles en la reputación de la empresa.

Por otra parte, analizando el resultado obtenido, se puede indicar que el desempeño de cada uno de los empleados de una empresa dependerá de la aplicación de verdaderos principios, valores y en explotar al máximo las propias capacidades, habilidades y conocimientos de modo que el conjunto de personas y entes a su alrededor pueda beneficiarse de 
ellas, lo que logrará que los integrantes de las empresas obtengan unos excelentes resultados en su desempeño laboral como hasta ahora lo son, movidos en conjunto por un interés común, el mismo que persigue la organización.

\section{Referencias Bibliográficas}

Aragón, Antonio (2004). Fundamentos de dirección y gestión de recursos humanos. Thomson Learning lbero. España.

Bentham, Jeremías (2000). Principios de la ciencia social o de las ciencias morales y políticas, ed. de Toribio Núñez, 2 vols., Imprenta de Bernardo Martín, Salamanca, 1821; reed. como La Ciencia Social Según los principios de Jeremías Bentham. Madrid: [s.n.], 1835 (Impr. Real).

Chiavenato, Idalberto (1998). Administración de Recursos Humanos. Segunda Edición. Editorial Mc Graw Hill Interamericana, S.A. Colombia.

Cortina, Adela (1998). Ética de la Empresa. Tercera Edición. Editorial Trotta, S.A. Madrid-España.

Cuesta F., Félix (1998). La Empresa Virtual. Editorial McGraw Hill Interamericana, Madrid-España.

Etkin, Jorge R. (1993). “La Doble Moral de las Organizaciones”. Editorial Mc Graw Hill Interamericana de España, S.A.

Fairbanks, Michael (1999). Arando en el Mar. Editorial Mc Graw Hill Interamericana, México.

Guédez, Víctor (2001). La Ética Gerencial. Editorial Planeta. Caracas, Venezuela.

García, Salvador y Dolan, Shimon (1997). "Dirección por Valores". Editorial Mc Graw Hill Interamericana de España.
Harbour, Jerry L. (1999). "Fundamentos de Medición del Desempeño en la Empresa". Ed. Panorama, México.

Leer, Anne (2000) "La Visión de los Líderes en la Era Digital”, México.

Montaner, Ramón (2001). "Dirigir con las Nuevas Tecnologías". Ed. Gestión 2000. Barcelona-España.

Mills, John (2008). "John Stuart Mill (1806 1873)" disponible en: http://www.utilitarianism.com/ jsmill.htm, consultado el 02 de feb. 2008.

Ortiz, José M. (1995). "La Hora de la Ética Empresarial”. Editorial Mc Graw Hill Interamericana de España.

Pérez, José A. (1998). "Liderazgo y Ética en la dirección de empresas". Ediciones Deusto, S.A.

Puchol, Luis (2005). "Dirección y Gestión de Recursos Humanos". Ediciones Díaz de Santos.

Siliceo A., Alfonso y Col. (1999). "Liderazgo, Valores y Cultura Organizacional”. Editorial Mc Graw Hill Interamericana Editores, S.A. México.

Silva, Neif y Espina, Jane (2006). "Ética Informática en la sociedad Actual". Revista Venezolana de Gerencia. Año 11 No. 36. Universidad del Zulia (LUZ).

Silva, Neif (2008). Cultura Informática en la gestión de actividades del profesor universitario. Trabajo de Ascenso. FCES. Universidad del Zulia (LUZ).

Stoner, James A.F. (1994). Administración. 5ta. Edición. Editorial Prentice - Hall Hispanoamericana S.A.

Werther, David (2001). Administración de Personal y Recursos Humanos. Editorial Mc Graw Hill Interamericana. 